\title{
EVOLUTION OF SUBSYSTEMS DURING COLLAPSE OF A CLUSTER
}

\author{
TOSHIO TSUCHIYA \\ Department of Astronomy, Kyoto University \\ Kyoto 606-01, Japan
}

Motivation: In the hierarchical clustering scenario, objects with smaller masses collapse and virialize earlier. I am interested in the effects of the existence of virialized subsystems on the structures of a collapsing larger mass object, and the fate of subsystems.

Comparison to previous works: Most work, both analytic and numerical, is concerned with the evolution of subsystems in a virialized cluster. Some numerical works have investigated dynamical effects in collapsing clusters, but these are usually not quite systematic.

Aim of this work: We try to study numerically the evolution of collapsing clusters with clumpy initial conditions. We make a systematic survey of two dimensional parameters: the number and the relative size of the subsystems. Simulations: Numerical N-body simulations are made with the GRAPE$3 \mathrm{~A}$ supercomputer. No cosmological expansion is included. Each subsystem is an equilibrium Plummer sphere. The initial radius of the cluster of the Plummer sphere is given by

$$
R_{0} \equiv 2 r_{\mathrm{v}}\left(\frac{M_{\text {total }}}{m}\right)^{\left(n_{\mathrm{k}}+5\right) / 6}=N_{\mathrm{c}}^{\left(n_{\mathrm{k}}+5\right) / 6}
$$

where $r_{\mathrm{v}}$ is the initial radius of the cluster and the subsystems; $M_{\text {total }}$ and $m$ are the masses of the cluster and the subsystems and $N_{\mathrm{c}}$ is the number of the subsystems.

Results: We obtained a condition for the subsystems to survive the first violent phase of a collapsing cluster;

$$
n_{\mathrm{k}} \geq-1, \quad \text { and } \quad N_{\mathrm{c}} \gg 1 .
$$

